\title{
Actualización en lupus neuro-psiquiátrico con énfasis en déficit cognitivo
}

\author{
TOMÁS LEÓN, CARLA HENRÍQUEZ \\ JORGE CALDERÓN, LORETO MASSARDO
}

\section{An update on neuropsychiatric lupus with emphasis in cognitive dysfunction}

Patients with systemic lupus erythematosus (SLE) suffer from a number of neuropsychiatric $(N P)$ symptoms throughout their disease affecting them both physically and psychologically. We review herein the nomenclature and case definitions for neuropsychiatric lupus syndromes proposed by the American College of Rheumatology in 1999. We emphasize cognitive dysfunction and discuss etiological hypotheses, especially those related to the presence of antineuronal autoantibodies.

(Rev Med Chile 2012; 140: 1333-1341).

Key words: Autoantibodies; Lupus erythematosus systemic; Neurobehavioral manifestations.

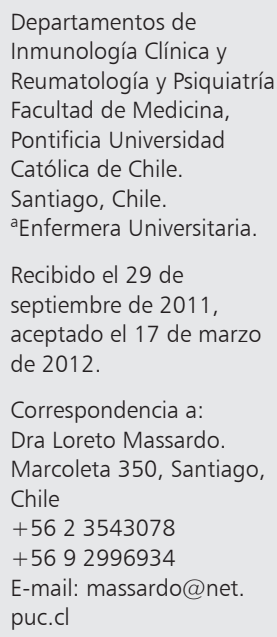

$\mathrm{E}$ 1 lupus eritematoso sistémico (LES) es una enfermedad multisistémica inflamatoria autoinmune de etiología desconocida, que afecta especialmente a mujeres entre los 15 y 50 años de edad. La supervivencia de los pacientes con LES ha mejorado notablemente en las últimas décadas ${ }^{1}$, poniendo de manifiesto la necesidad de comprender mejor los síntomas neuro-psiquiátricos (NP) del LES, entre los que se encuentra el déficit cognitivo, ya que favorece el sufrimiento del enfermo y su entorno ${ }^{2,3}$. El objetivo de esta revisión es realizar una puesta al día en diagnóstico y etiopatogenia del lupus neuro-psiquiátrico (LESNP), con énfasis en el déficit cognitivo.

El LES es considerado el prototipo de enfermedad autoinmune, se caracteriza por la producción de una variedad de autoanticuerpos y depósito de complejos inmunes que fijan complemento, lo que resulta en inflamación y daño tisular. Los tejidos afectados más estudiados son la piel y el riñón, pero el LES tiene un gran espectro de otras manifestaciones clínicas, como las neurológicas y psiquiátricas ${ }^{4}$. La presencia de ciertos autoanticuerpos en el suero se asocia a subtipos clínicos de enfermedad, sugiriendo que estos autoanticuerpos participarían en la patogénesis. Son ejemplos de posibles asociaciones: anticuerpos anti-DNA con nefritis lúpica; anti-Ro con bloqueo cardiaco fetal, foto-sensibilidad y lesiones cutáneas; antifosfolípidos (AFL) con trombosis y compromiso neurológico; $\mathrm{y}$ anti proteína $\mathrm{P}$ ribosomal (anti-P) con psicosis lúpica ${ }^{3}$.

Las manifestaciones del LESNP son bien reconocidas. Hace más de cien años, el Dr. Moritz Kaposi describió que "cambios mentales" podían acompañar el curso clínico de los pacientes con LES. Tradicionalmente, se han dividido en focales y generalizadas, siendo las primeras relacionadas con lesiones isquémicas por vasculopatía no necesariamente inflamatoria o trombosis, como ocurre con los estados de hipercoagulabilidad, y las segundas a la acción de diferentes tipos de autoanticuerpos antineuronales y otros ${ }^{5-7}$. Los estudios iniciales reportaron una gran variabilidad en la prevalencia de las manifestaciones psiquiátricas del lupus. Por ejemplo, Wekking revisó 21 estudios realizados entre los años 1969 y 1991 y encontró que la prevalencia variaba entre 17\% y $71 \%$. El trastorno observado con mayor frecuencia fue la depresión, que se reportó entre $2 \%$ y $54 \%$ de los enfermos. Estas diferencias se atribuyeron a la diversidad en los instrumentos utilizados para la detección de depresión ${ }^{8}$.

Para dirimir la controversia y aunar criterios 
de estudio, en el año 1999 el American College of Rheumatology (ACR) encargó a una comisión ad hoc de expertos el desarrollo de una nomenclatura que uniformara y estandarizara la descripción de los síndromes NP en lupus. El comité describió un total de 19 síndromes psiquiátricos y neurológicos del sistema nervioso central (SNC), periférico y autónomo que se observan en pacientes con lupus. Estos son: meningitis aséptica, enfermedad cerebro-vascular, síndrome desmielinizante, cefalea, corea, mielopatía, convulsiones, delirium, trastorno de ansiedad, déficit cognitivo, trastorno del ánimo, psicosis, lesión del nervio periférico, el síndrome Guillain-Barré, trastornos autonómicos, mononeuropatía, miastenia gravis, neuropatía craneal y plexopatía ${ }^{4}$. Sin embargo, aun usando esta clasificación común que unifica y define criterios de clínica, laboratorio e imágenes o pruebas psicológicas para cada síndrome, no se dio respuesta a cuál es la prevalencia del LESNP, ya que esta varió de $37 \%$ a $91 \%$ en 6 estudios que la utilizaron, incluyendo el nuestro ${ }^{9-14}$. Entre todas las manifestaciones, los trastornos del ánimo y ansiosos y el déficit cognitivo fueron los más comunes (Tabla 1). En el año 2008, Sánchez-Guerrero et al, propusieron un nuevo esquema de clasificación pato-anatómico, considerando la variabilidad clínica del LESNP según las posibles etiopatoge-

\section{Tabla 1. Frecuencia en porcentajes de las manifestaciones de LESNP en 6 estudios que utilizaron los 19 criterios ACR 1999}

\begin{tabular}{|c|c|c|c|c|c|c|}
\hline Manifestaciones de LESNP & Finlandia & Texas & $\begin{array}{c}\text { Londres y } \\
\text { Cagliari }\end{array}$ & Canadá & Alburquerque & Santiago \\
\hline Referencia & 9 & 10 & 12 & 13 & 11 & 14 \\
\hline Numero de pacientes estudiados & 46 & 128 & 323 & 111 & 75 & 83 \\
\hline Años de duración de lupus & 14 & 8 & 11 & 10. & 8 & 5 \\
\hline Frecuencia de LUPUS NP en \% & 91 & 80 & 57 & 37 & 95 & 42 \\
\hline $\begin{array}{l}\text { Frecuencia de cada } \\
\text { manifestación en } \%\end{array}$ & $\%$ & $\%$ & $\%$ & $\%$ & $\%$ & $\%$ \\
\hline Trastornos del ánimo & 44 & 51 & 17 & 16 & 57 & 29 \\
\hline Trastornos ansiosos & 13 & 24 & 7 & 1 & 21 & 16 \\
\hline Disfunción cognitiva & 80 & 79 & 11 & 3 & 55 & 7 * \\
\hline Estado confusional agudo & 7 & 0 & 4 & 5 & 35 & 6 \\
\hline Cefalea lúpica & 54 & 57 & 24 & 28 & 72 & 3 \\
\hline Enfermedad cerebro-vascular & 15 & 2 & 18 & 5 & 12 & 5 \\
\hline Psicosis & 0 & 5 & 8 & 3 & 12 & 3 \\
\hline Síndrome desmielinizante & 2 & 0 & 1 & 3 & 4 & 1 \\
\hline Mononeuropatia & 0 & 8 & 2 & 0 & 1 & 1 \\
\hline Meningitis aséptica & 2 & 0 & 0 & 1 & 1 & 0 \\
\hline Convulsiones & 9 & 16 & 8 & 2 & 1 & 0 \\
\hline Corea & 2 & 1 & 1 & 0 & 51 & 0 \\
\hline Mielopatía & 0 & 4 & 1 & 1 & 7 & 0 \\
\hline Miastenia gravis & 2 & 0 & 5 & 0 & 1 & 0 \\
\hline Neuropatía par craneano & 7 & 2 & 2 & 4 & 0 & 0 \\
\hline Polineuropatía & 28 & 22 & 3 & 2 & 1 & 0 \\
\hline Guillain Barré & 0 & 0 & 1 & 0 & 0 & 0 \\
\hline
\end{tabular}

*En el estudio de Santiago 2011 se consideró como déficit cognitivo sólo a los casos graves. **No se encontró plexopatía ni trastorno autonómico en ninguno de los 6 estudios. 
nias, en el que se agrupan las manifestaciones en: 1) Centrales del parénquima cerebral (deterioro cognitivo no debido a micro infartos, trastornos del ánimo, psicosis, trastorno "confusional" agudo y cefalea no vascular): este grupo es el predominante en los estudios clínicos y su etiopatogenia estaría potencialmente ligada a los autoanticuerpos; 2) Vasculares: segundo en frecuencia y con una patogenia atribuible a lesiones isquémicas por vasculopatía (no necesariamente inflamatoria) y/o trombosis; 3 ) Del sistema nervioso periférico: es el menos frecuente, su patogenia es mixta por autoanticuerpos y por alteraciones vasculares ${ }^{15}$.

En nuestro país, en el año 1985 Grisanti et al describieron 20 pacientes con manifestaciones de LESNP, planteando una diferencia entre las manifestaciones vasculares y las difusas ${ }^{16}$. En 1994, nuestro grupo estudió la supervivencia de 218 enfermos con LES, con 5 años de duración de la enfermedad en promedio, 20\% había presentado convulsiones o psicosis ${ }^{17}$. Recientemente, describimos una prevalencia de $42 \%$ de LESNP en 83 mujeres con LES (Tabla 1), 21\% presentaba depresión mayor no relacionada con la actividad lúpica ${ }^{14}$.

Otro motivo de controversia es la atribución de manifestaciones NP al LES. Según Hanly et al, las manifestaciones NP atribuibles a LES como enfermedad autoinmune serían sólo de $31 \% 0^{7,18,19}$. En un extremo se encuentran las convulsiones y la psicosis lúpica, que son manifestaciones claramente atribuibles al LES, tanto que forman parte de los criterios de clasificación diagnóstica de LES del $\mathrm{ACR}^{20}$, y si bien son poco frecuentes, se suelen presentar con enfermedad activa ${ }^{21,22}$. Al contrario, los síndromes NP como trastornos de ánimo y ansiosos, cefalea, y el déficit en el rendimiento cognitivo son atribuibles al lupus con más dificultad, pues pudiera ser coincidencia de dos enfermedades comunes, y además porque se observan con independencia de la actividad de la enfermedad ${ }^{7,9,10,14,23,24}$.

\section{Déficit cognitivo en LES}

Los pacientes con LES con frecuencia se quejan de "dificultades en la memoria". Sin embargo, en estos pacientes aún no está claro que las dificultades se centren en la memoria, sino en procesos de atención y concentración, síntomas que se desarrollan en forma insidiosa e independiente de la actividad de la enfermedad o del uso de medicamentos y no pueden explicarse únicamente sobre la base de, por ejemplo, la coexistencia de AFL en suero ${ }^{25}$. El ACR define el déficit cognitivo como un déficit en uno o más de los siguientes dominios de la cognición: atención simple, atención compleja, memoria, procesamiento visual, lenguaje, razonamiento y resolución de problemas, y función ejecutiva ${ }^{4}$. Mikdashi et $\mathrm{al}^{26}$ en el año 2007 hicieron una propuesta de criterios de medición neuro-cognitiva utilizables en evaluación de la respuesta a intervenciones terapéuticas. La frecuencia de déficit cognitivo en LES varió entre $27 \%$ y $61 \%$ en 25 estudios, posiblemente por el uso de diferentes escalas de medición, definiciones de déficit y selección de pacientes y controles. Petri et al demostraron que el trastorno cognitivo se presentó en el primer año del lupus en 111 enfermos, comparado con 79 sanos, ajustado por edad, sexo, educación; lo que no estuvo influenciado por el uso de prednisona ${ }^{27}$, pero sí es explicable por la presencia de depresión ${ }^{28}$. Aunque varios estudios de déficit cognitivo en LES no revelan un patrón definido, éstos sugieren que el déficit abarca dificultades en pruebas que evalúan los dominios de atención, memoria reciente, velocidad de procesamiento de la información y memoria de trabajo, y procesamiento espacial ${ }^{29,30}$. Ainiala et al encontraron déficit cognitivo significativo en 11 de 46 LES versus 2 de 46 controles pareados por edad y sexo; el déficit cognitivo era más pronunciado en velocidad de procesamiento de la información, atención compleja y memoria ${ }^{9}$, patrón similar al de la esclerosis múltiple ${ }^{31}$. Se ha especulado que el déficit cognitivo en LES tendría un patrón de tipo subcortical, más parecido a la enfermedad de Huntington que al patrón cortical de la Enfermedad de Alzheimer ${ }^{29}$.

\section{Etiopatogenia del LESNP}

\section{Papel de la barrera hemato-encefálica}

El origen de muchas de las manifestaciones del LESNP es desconocido, aunque probablemente es multifactorial. Los mecanismos inmunopatogénicos y de autoanticuerpos pueden tener un papel relevante, en especial aquellos en los que se involucre daño a la barrera hemato-encefálica (BHE). Las células endoteliales de la microcirculación del cerebro forman una barrera impenetrable para el 
paso de anticuerpos; sin embargo, la integridad de la BHE puede ser afectada como resultado de infecciones (lipopolisacáridos), hipertensión o conductas como el estrés (epinefrina) o consumo de tabaco ${ }^{32,33}$. Esta permeabilidad pudiera producirse en áreas localizadas en el cerebro, y la manifestación depender del área expuesta a la acción de los anticuerpos ${ }^{25}$. Estos mecanismos pueden determinar la penetración crónica de autoanticuerpos mediante pequeñas filtraciones, $y$ explicar que el déficit cognitivo se haya observado en momentos en que el LES parece clínicamente inactivo.

\section{Autoanticuerpos en LESNP}

Se han descrito numerosos autoanticuerpos en el suero de pacientes con LES, para algunos se conoce su antígeno y su posible mecanismo de acción ${ }^{3}$. En el LESNP, sólo unos pocos se han asociado con manifestaciones clínicas, estos tres por su importancia se presentan a continuación:

AFL: cuyo blanco son autoantígenos dependientes de fosfolípidos. Están presentes en alrededor de $20 \%$ a $40 \%$ de los pacientes con LES, aunque menos de la mitad presenta clínica de "Síndrome antifosfolipido"34. En el cerebro, se han asociado con daño focal cerebral secundario a trombosis, déficit cognitivo ${ }^{35-37}$, demencia, convulsiones, corea y otras manifestaciones del SNC como mielitis transversa. Es interesante que no todas estén relacionadas claramente con trombosis $^{35-37}$.

Anti-NMDAR: los anticuerpos contra la subunidad NR2 del receptor de glutamato N-metil D aspartato (NMDAR), reconocido por un subgrupo de anticuerpos anti-DNA. La frecuencia va de $10 \%$ a $30 \%{ }^{38-40}$. Juegan un papel en alteraciones conductuales relacionadas con fallas de memoria en modelos animales ${ }^{41}$, sin embargo, no se dispone hasta ahora de información que apoye esta hipótesis en humanos ${ }^{38,40,42}$. Su presencia en el líquido céfalo raquídeo (LCR) sería más importante en términos etiopatogénicos que en el suero ${ }^{43}$.

Anti-P: dirigidos contra tres antígenos ribosomales: P0, P1 y P2 que comparten un epítopo común de 22 aminoácidos en su carboxilo terminal. Se encuentran de $5 \%$ a $13 \%$ de los pacientes con LES. Son específicos de LES, y se han relacionado con LESNP en globo (psicosis lúpica y trastornos depresivos), pero con una potencia predictora débil $^{21,34,44-48}$. Recientemente, Matus et al, trabajando en nuestro laboratorio, identificaron un autoanticuerpo proveniente de una paciente con psicosis lúpica dirigido contra un antígeno $\mathrm{P}$ ubicado en la superficie neuronal (llamado NSPA por sus siglas en inglés), reconocido por autoanticuerpos anti- $\mathrm{P}^{49}$.

Otros autoanticuerpos: varios otros fueron descritos entre los años 2007 y 2011, seis de ellos se revisan a continuación: Anticuerpo anti inhibidor alfa de la disociación de la guanidina difosfato, abundante en las neuronas y necesario para la liberación de neurotrasmisores, al que se le atribuye una relación con la psicosis lúpica ${ }^{50}$. Anticuerpo antigangliósido M1, que fue relacionado con déficit cognitivo en LES infantil ${ }^{51}$. Anticuerpos antineuronales, cuyo antígeno blanco son proteínas de superficie neuronales, se describieron en 13 de 17 enfermos con LES con compromiso del SNC versus 1 de 27 LES sin compromiso del $\mathrm{SNC}^{52}$. Anticuerpos antitubulina fueron descritos en 50\% de LESNP grave versus 20\% de LESNP $\operatorname{leve}^{53}$. Anticuerpos IgG dirigidos contra antígenos cerebrales contenidos en los microtúbulos neuronales (MAP-2B, triosafosfato isomerasa y septina 7) fueron encontrados en el suero de 16 sujetos con LESNP ${ }^{54}$. Niveles elevados del antígeno del anticuerpo antineurofilamento internectina, que se ha asociado con daño cognitivo experimental en ratones, fue descrito en pacientes LESNP ${ }^{55}$.

\section{Otros factores en la etiología}

Además, los síntomas de LESNP se han asociado con la presencia de niveles elevados de proteínas inflamatorias interferón e interleuquina 6 (IL-6) en suero y en $\mathrm{LCR}^{56-58}$. Su presencia en LCR podría significar un peor pronóstico del los síntomas $\mathrm{NP}^{59}$. Algunos pacientes con LESNP presentan niveles significativamente más altos del inhibidor de activación del plasminógeno en LCR, lo que pudiera alterar la fibrinólisis y de este modo aumentar el daño vascular ${ }^{60}$.

Por otro lado, se ha buscado una etiología viral que pudiese explicar el comienzo de la reacción inflamatoria, por ejemplo, una asociación se describió entre niveles de IgM contra rubéola en sangre con psicosis o depresión ${ }^{61}$. Niveles elevados de un "factor neurotrópico cerebral" en el suero se asociarían con síntomas psiquiátricos, pero no neurológicos, en pacientes LESNP, y que serían 
concordantes con la magnitud de la enfermedad ${ }^{62}$. Otro hallazgo son niveles altos de S100B, una proteína relacionada al metabolismo del calcio, en sangre y LCR de pacientes con LESNP, especialmente en aquellos con daño cerebral orgánico, accidente vascular encefálico, convulsiones o psicosis; sin conocerse su función en la etiopatogenia de estas manifestaciones ${ }^{63}$. También se encontró que al existir daño en la BHE, el riesgo de psicosis post-corticoides aumentaba significativamente ${ }^{33}$.

\section{Exámenes diagnósticos en suero}

En Chile, en laboratorios especializados es posible pesquisar anticoagulante lúpico por técnicas de coagulación, medir niveles de anticardiolipinas y de anti-beta 2 glicoproteina I por técnicas ELISA comercial. Anti-P se mide por ELISA comercial que se confirma por inmunoblot y anti-NSPA se detecta por inmunoblot. Sin embargo, la correlación con los hallazgos clínicos NP en el paciente individual y la interpretación son difíciles ${ }^{48}$. Por otra parte, hay diferencias entre los niveles de anticuerpos de una misma persona según el sitio de medición: suero o $\mathrm{LCR}^{43,64}$. Por otro lado, la cuantificación de una elevación de IL-6 en LCR podría ser diagnóstica de LESNP, con una sensibilidad de $87 \%$ y una especificidad de $93 \%$, una vez descartada la posibilidad de una meningitis ${ }^{58,65}$.

\section{Imágenes diagnósticas}

La principal finalidad de los estudios diagnósticos de imágenes en LESNP es que permiten establecer el diagnóstico diferencial con otras lesiones que potencialmente afectan al cerebro, como abscesos, hematomas o accidentes cerebrovasculares, esto es especialmente importante en pacientes en uso de inmunosupresores, con trastornos de coagulación o trombocitopenias o hipertensión. Por largo tiempo se han buscado patrones que permitan diferenciar pacientes con LESNP de los pacientes sin LESNP; en el LESNP se ha descrito que la resonancia nuclear magnética (RNM) es normal o se observan imágenes focales, pequeñas, hiper intensas en T2 en la sustancia blanca periventricular y subcortical, ubicadas usualmente en la región frontal, con mala correlación clínica, ya que también se observan en LES no NP. La combinación de RNM con estudios funcionales de tomografía computarizada por emisión de fotones individuales o SPECT pareciera tener mayor sensibilidad para detectar compro- miso del SNC por lupus, tanto en la forma difusa como en la focal ${ }^{66}$. En pacientes con LESNP se encontró una baja perfusión en el lóbulo frontal al utilizar SPECT y la intensidad de esta lesión se correlacionó con mayor déficit cognitivo ${ }^{67}$. En lo que respecta a la RNM funcional se ha observado una mayor activación frontoparietal en tareas de memoria, posiblemente por reclutar más vías neuronales por defecto de las originales ${ }^{68}$, y alteraciones en la sustancia blanca del cuerpo calloso en pacientes LESNP ${ }^{69,70}$ o un adelgazamiento de la sustancia gris cortical y subcortical ${ }^{71,72}$. No se encontró asociación en la disfunción endotelial cerebral cuantificada a través de Eco Dopplerer,73, ni en histograma de transferencia magnética ${ }^{74}$.

\section{Recomendaciones de terapia}

Como se establece en las guías del año 2010 de la liga europea "The European League Against Rheumatism" las manifestaciones NP en LES debieran ser primeramente evaluadas y tratadas como en los pacientes sin LES. Una vez realizada la exclusión de las causas no relacionadas con LESNP, el uso de corticoesteroides e inmunosupresores (como azatioprina o ciclofosfamida) están indicados en las manifestaciones autoinmunes inflamatorias de LESNP, como son el estado confusional agudo, meningitis aséptica, mielitis, psicosis, y neuropatías de par craneano y las periféricas. La terapia anticoagulante o antiplaquetaria está indicada para tratamiento y prevención de la enfermedad cerebro-vascular relacionada con AFL. Sin embargo, se carece de un buen nivel de evidencia de las recomendaciones de terapia en LESNP $^{75}$.

\section{Conclusiones}

Las manifestaciones del LESNP comprometen la calidad de vida, el empleo y posiblemente la supervivencia de pacientes con $\mathrm{LES}^{76}$. El déficit cognitivo es una manifestación frecuente y posiblemente menos reconocida en nuestro medio, y es importante discriminar si éste se produce como un epifenómeno de otras variables como depresión y ansiedad, uso de fármacos y gravedad de la enfermedad, o si más bien responde a un efecto directo del LES sobre estructuras cerebrales a través de los 
mecanismos etiopatogénicos descritos. En otras palabras, se requiere diferenciar las manifestaciones propias del LES de los síntomas y signos dados por el tratamiento o sus complicaciones, o por las situaciones psicosociales que acompañan el tener una enfermedad inflamatoria crónica grave. En los mecanismos etiopatogénicos se postula que un daño en la BHE permite que se pongan en contacto autoanticuerpos circulantes dirigidos contra antígenos neuronales con sus antígenos, lo que pudiera desencadenar procesos inflamatorios en el SNC que se traduzcan en síntomas. Actualmente, los autoanticuerpos que están siendo estudiados en déficit cognitivo son los AFL, anti MMDAR y el anti-P/ NSPA. Estimular la investigación en LESNP permitirá un mejor diagnóstico, establecer factores de riesgo y de pronósticos y esperamos que esto conduzca a un tratamiento más adecuado de estos pacientes.

Agradecimientos: Manuscrito financiado por proyectos FONDECYT \#1085283 y \#1110849.

\section{Referencias}

1. Vasudevan A, Krishnamurthy AN. Changing worldwide epidemiology of systemic lupus erythematosus. Rheumatic Disease Clin North Am 2010; 36 (1): 1-13.

2. Karassa FB, Ioannidis JP, Boki KA, Touloumi G, Argyropoulou MI, Strigaris KA, et al. Predictors of clinical outcome and radiologic progression in patients with neuropsychiatric manifestations of systemic lupus erythematosus. Am J Med 2000; 109 (8): 628-34.

3. Wallace DJ, Hahn BH. Dubois`Lupus Eryhtematosus. 7 ed. Philadelphia: Lippincott Williams \& Wilkins; 2007.

4. The American College of Rheumatology nomenclature and case definitions for neuropsychiatric lupus syndromes. Arthritis Rheum 1999; 42 (4): 599-608.

5. Sergent JS, Lockshin MD, Klempner MS, Lipsky BA. Central nervous system disease in systemic lupus erythematosus. Therapy and prognosis. Am J Med 1975; 58 (5): 644-54

6. Boumpas DT, Austin HA, 3rd, Fessler BJ, Balow JE, Klippel JH, Lockshin MD. Systemic lupus erythematosus: emerging concepts. Part 1: Renal, neuropsychiatric, cardiovascular, pulmonary, and hematologic disease. Ann Intern Med 1995; 122 (12): 940-50.

7. Hanly JG, Harrison MJ. Management of neuropsychiatric lupus. Best Pract Res Clin Rheumatol 2005; 19 (5): 799-821.
8. Wekking EM. Psychiatric symptoms in systemic lupus erythematosus: an update. Psychosomatic Med 1993; 55 (2): 219-28.

9. Ainiala H, Hietaharju A, Loukkola J, Peltola J, Korpela M, Metsanoja R, et al. Validity of the new American College of Rheumatology criteria for neuropsychiatric lupus syndromes: a population-based evaluation. Arthritis Rheum 2001; 45 (5): 419-23.

10. Brey RL, Holliday SL, Saklad AR, Navarrete MG, Hermosillo-Romo D, Stallworth CL, et al. Neuropsychiatric syndromes in lupus: prevalence using standardized definitions. Neurology 2002; 58 (8): 1214-20.

11. Sibbitt WL, Jr., Brandt JR, Johnson CR, Maldonado ME, Patel SR, Ford CC, et al. The incidence and prevalence of neuropsychiatric syndromes in pediatric onset systemic lupus erythematosus. J Rheumatol 2002; 29 (7): 1536-42.

12. Sanna G, Bertolaccini ML, Cuadrado MJ, Laing H, Khamashta MA, Mathieu A, et al. Neuropsychiatric manifestations in systemic lupus erythematosus: prevalence and association with antiphospholipid antibodies. J Rheumatol 2003; 30 (5): 985-92.

13. Hanly JG, McCurdy G, Fougere L, Douglas JA, Thompson K. Neuropsychiatric events in systemic lupus erythematosus: attribution and clinical significance. J Rheumatol 2004; 31 (11): 2156-62.

14. Jarpa E, Babul M, Calderón J, González M, Martínez M, Bravo-Zehnder M, et al. Common Mental Disorders And Psychological Distress In Systemic Lupus Erythematosus Are Not Associated With Disease Activity. Lupus 2011; 20 (1): 58-66.

15. Sánchez-Guerrero J, Aranow C, Mackay M, Volpe B, Diamond B. Neuropsychiatric systemic lupus erythematosus reconsidered. Nat Clin Pract Rheumatol 2008; $4(3): 112-3$.

16. Grisanti MA, Vergara F, Cartier L, Guzmán L. Involvement of central nervous system in systemic lupus erythematosus. Experience with 20 patients. Rev Med Chile 1985; 113 (12): 1194-202.

17. Massardo L, Martínez ME, Jacobelli S, Villarroel L, Rosenberg H, Rivero S. Survival of Chilean patients with systemic lupus erythematosus. Semin Arthritis Rheum 1994; 24 (1): 1-11.

18. Hanly JG, Urowitz MB, Sánchez-Guerrero J, Bae SC, Gordon C, Wallace DJ, et al. Neuropsychiatric events at the time of diagnosis of systemic lupus erythematosus: an international inception cohort study. Arthritis Rheum 2007; 56 (1): 265-73.

19. Hanly JG, Su LI, Farewell V, McCurdy G, Fougere L, Thompson K. Prospective Study of Neuropsychiatric Events in Systemic Lupus Erythematosus. J Rheumatol 2009; 36 (7): 1449-59. 
20. Hochberg M, Silman A, Smolen J, Weinblatt M, Weisman MH. Rheumatology. 4 ed. Philadelphia; 2008.

21. Pego-Reigosa JM, Isenberg DA. Psychosis due to systemic lupus erythematosus: characteristics and long-term outcome of this rare manifestation of the disease. Rheumatology (Oxford) 2008; 47 (10): 1498-502.

22. Cantú-Brito C, Baizabal-Carvallo JF, Alonso-Juárez M, García-Ramos G. The clinical significance of microembolic signals in patients with systemic lupus erythematosus. Neurol Res 2010; 32 (2): 134-8.

23. Hanly JG, Fisk JD, Sherwood G, Jones E, Jones JV, Eastwood B. Cognitive impairment in patients with systemic lupus erythematosus. J Rheumatol 1992; 19 (4): 562-7.

24. Kozora E, Thompson LL, West SG, Kotzin BL. Analysis of cognitive and psychological deficits in systemic lupus erythematosus patients without overt central nervous system disease. Arthritis Rheum 1996; 39 (12): 2035-45.

25. Aranow C, Diamond B, Mackay M. Glutamate receptor biology and its clinical significance in neuropsychiatric systemic lupus erythematosus. Rheum Dis Clin North Am 2010; 36 (1): 187-201, x-xi.

26. Mikdashi JA, Esdaile JM, Alarcon GS, Crofford L, Fessler BJ, Shanberg L, et al. Proposed response criteria for neurocognitive impairment in systemic lupus erythematosus clinical trials. Lupus 2007; 16 (6): 418-25.

27. Petri M, Naqibuddin M, Carson KA, Sampedro M, Wallace DJ, Weisman $\mathrm{MH}$, et al. Cognitive function in a systemic lupus erythematosus inception cohort. J Rheumatol 2008; 35 (9): 1776-81.

28. Petri M, Naqibuddin M, Carson KA, Wallace DJ, Weisman MH, Holliday SL, et al. Depression and Cognitive Impairment in Newly Diagnosed Systemic Lupus Erythematosus. J Rheumatol 2010; 37 (10): 2032-8.

29. Benedict RH, Shucard JL, Zivadinov R, Shucard DW. Neuropsychological impairment in systemic lupus erythematosus: a comparison with multiple sclerosis. Neuropsychol Rev 2008; 18 (2): 149-66.

30. Hanly J, Omisade A, Su L, Farewell V, Fisk JD. Assessment of cognitive function in systemic lupus erythematosus, rheumatoid arthritis, and multiple sclerosis by computerized neuropsychological tests. Arthritis Rheuma 2010; 62 (5): 1478-86.

31. Loukkola J, Laine M, Ainiala H, Peltola J, Metsanoja R, Auvinen A, et al. Cognitive impairment in systemic lupus erythematosus and neuropsychiatric systemic lupus erythematosus: a population-based neuropsychological study. J Clin Exp Neuropsychol 2003; 25 (1): 145-51.

32. Abbott NJ, Mendonca LL, Dolman DE. The blood-brain barrier in systemic lupus erythematosus. Lupus 2003; 12 (12): 908-15.

33. Nishimura K, Harigai M, Omori M, Sato E, Hara M.
Blood-brain barrier damage as a risk factor for corticosteroid-induced psychiatric disorders in systemic lupus erythematosus. Psychoneuroendocrinology 2008; 33 (3): 395-403.

34. Reeves WE, Li Y, Zhuang H. Autoantibodies in systemic lupus erythematosus. In: Hochberg MC, A.J. S, Smolens JS, Weinblat ME, Weisman MH, editors. Rheumatology. Fifth ed. Philadelphia: Mosby Elsevier; 2010. p. 12791287.

35. Denburg SD, Denburg JA. Cognitive dysfunction and antiphospholipid antibodies in systemic lupus erythematosus. Lupus 2003; 12 (12): 883-90.

36. Cervera R, Piette JC, Font J, Khamashta MA, Shoenfeld Y, Camps MT, et al. Antiphospholipid syndrome: clinical and immunologic manifestations and patterns of disease expression in a cohort of 1,000 patients. Arthritis Rheum 2002; 46 (4): 1019-27.

37. Khamashta MA, Pierangeli S, Harris NE. Antiphospholipid syndroem: overview of pathogenesis, diagnosis and management. Philadelphia: Mosby Elsevier; 2010.

38. Hanly JG, Robichaud J, Fisk JD. Anti-NR2 glutamate receptor antibodies and cognitive function in systemic lupus erythematosus. J Rheumatol 2006; 33 (8): 1553-8.

39. Steup-Beekman G, Steens S, van Buchem M, Huizinga T. Anti-NMDA receptor autoantibodies in patients with systemic lupus erythematosus and their first-degree relatives. Lupus 2007; 16 (5): 329-34.

40. Lapteva L, Nowak M, Yarboro CH, Takada K, RoebuckSpencer T, Weickert T, et al. Anti-N-methyl-D-aspartate receptor antibodies, cognitive dysfunction, and depression in systemic lupus erythematosus. Arthritis Rheum 2006; 54 (8): 2505-14.

41. DeGiorgio LA, Konstantinov KN, Lee SC, Hardin JA, Volpe BT, Diamond B. A subset of lupus anti-DNA antibodies cross-reacts with the NR2 glutamate receptor in systemic lupus erythematosus. Nat Med 2001; 7 (11): 1189-93.

42. Huerta PT, Kowal C, DeGiorgio LA, Volpe BT, Diamond B. Immunity and behavior: antibodies alter emotion. Proc Natl Acad Sci U S A 2006; 103 (3): 678-83.

43. Arinuma Y, Yanagida T, Hirohata S. Association of cerebrospinal fluid anti-NR2 glutamate receptor antibodies with diffuse neuropsychiatric systemic lupus erythematosus. Arthritis Rheuma 2008; 58 (4): 1130-5.

44. Elkon KB, Bonfa E, Brot N. Antiribosomal antibodies in systemic lupus erythematosus. Rheum Dis Clin North Am 1992; 18 (2): 377-90.

45. Massardo L, Burgos P, Martínez ME, Pérez R, Calvo M, Barros J, et al. Antiribosomal $\mathrm{P}$ protein antibodies in Chilean SLE patients: no association with renal disease. Lupus 2002; 11 (6): 379-83. 
46. Diamond B, Kowal C, Huerta PT, Aranow C, Mackay M, DeGiorgio LA, et al. Immunity and acquired alterations in cognition and emotion: lessons from SLE. Adv Immunol 2006; 89: 289-320.

47. Ghirardello A, Briani C, Lucchetta M, Doria A. Antiribosomal $\mathrm{P}$ protein antibodies and neuropsychiatric systemic lupus erythematosus: cross-sectional vs prospective studies. Lupus 2010; 19 (6): 771-3.

48. Karassa FB, Afeltra A, Ambrozic A, Chang DM, De Keyser F, Doria A, et al. Accuracy of anti-ribosomal P protein antibody testing for the diagnosis of neuropsychiatric systemic lupus erythematosus: an international meta-analysis. Arthritis Rheuma 2006; 54 (1): 312-24.

49. Matus S, Burgos PV, Bravo-Zehnder M, Kraft R, Porras $\mathrm{OH}$, Farias $\mathrm{P}$, et al. Antiribosomal-P autoantibodies from psychiatric lupus target a novel neuronal surface protein causing calcium influx and apoptosis. J Exp Med 2007; 204 (13): 3221-34.

50. Kimura A, Kanoh Y, Sakurai T, Koumura A, Yamada M, Hayashi Y, et al. Antibodies in patients with neuropsychiatric systemic lupus erythematosus. Neurology 2010; 74 (17): 1372-9.

51. Mostafa GA, Ibrahim DH, Shehab AA, Mohammed AK. The role of measurement of serum autoantibodies in prediction of pediatric neuropsychiatric systemic lupus erythematosus. J Neuroimmunol 2010; 227 (1-2): 195201.

52. Kang EH, Shen GQ, Morris R, Metzger A, Lee EY, Lee YJ, et al. Flow cytometric assessment of anti-neuronal antibodies in central nervous system involvement of systemic lupus erythematosus and other autoimmune diseases. Lupus 2008; 17 (1): 21-5.

53. Ndhlovu M, Preuss BE, Dengjel J, Stevanovic S, Weiner $\mathrm{SM}$, Klein R. Identification of $\alpha$-tubulin as an autoantigen recognized by sera from patients with neuropsychiatric systemic lupus erythematosus. Brain, Behavior, and Immunity 2011; 25 (2): 279-85.

54. Lefranc D, Launay D, Dubucquoi S, de Seze J, Dussart $\mathrm{P}$, Vermersch $\mathrm{M}$, et al. Characterization of discriminant human brain antigenic targets in neuropsychiatric systemic lupus erythematosus using an immunoproteomic approach. Arthritis Rheum 2007; 56 (10): 3420-32.

55. Lu XY, Chen XX, Huang LD, Zhu CQ, Gu YY, Ye S. Antialpha-internexin autoantibody from neuropsychiatric lupus induce cognitive damage via inhibiting axonal elongation and promote neuron apoptosis. PloS one. 2010; 5 (6): 11124.

56. Fragoso-Loyo HE, Richaud-Patin Y, Orozco-Narváez A, Dávila-Maldonado L, Atisha-Fregoso Y, Llorente L, et al. Interleukin-6 and chemokines in the neuropsychiatric manifestations of systemic lupus erythematosus. Arthri- tis Rheuma 2007; 56 (4): 1242-1250.

57. Trysberg E, Tarkowski A. Cerebral inflammation and degeneration in systemic lupus erythematosus. Curr Opin Rheumatol 2004; 16 (5): 527-33.

58. Horai Y, Watanabe H, Miyamura T, Takahama S, Hirata A, Nakamura M, et al. Clinical analysis of cerebrospinal fluid interleukin-6 in neuropsychiatric systemic lupus erythematosus. Fukuoka Igaku Zasshi 2010; 101 (2): 34-40.

59. Stojanovich L, Smiljanich-Miljkovich D, Omdal R, Sakic B. Neuropsychiatric lupus and association with cerebrospinal fluid immunoglobulins: a pilot study. Isr Med Assoc J 2009; 11 (6): 359-62.

60. Kwiecinski J, Kłak M, Trysberg E, Blennow K, Tarkowski A, Jin T. Relationship between elevated cerebrospinal fluid levels of plasminogen activator inhibitor 1 and neuronal destruction in patients with neuropsychiatric systemic lupus erythematosus. Arthritis Rheuma 2009; 60 (7): 2094-101.

61. Zandman-Goddard G, Berkun Y, Barzilai O, Boaz M, Ram M, Anaya JM, et al. Neuropsychiatric lupus and infectious triggers. Lupus 2008; 17 (5): 380-4.

62. Ikenouchi-Sugita A, Yoshimura R, Okamoto T, UmeneNakano W, Ueda N, Hori H, et al. Serum brain-derived neurotrophic factor levels as a novel biological marker for the activities of psychiatric symptoms in systemic lupus erythematosus. World J Biol Psychiatry 2010; 11 (2): 121-8.

63. Yang XY, Lin J, Lu XY, Zhao XY. Expression of S100B protein levels in serum and cerebrospinal fluid with different forms of neuropsychiatric systemic lupus erythematosus. Clin Rheuma 2008; 27 (3): 353-7.

64. Fragoso-Loyo H, Cabiedes J, Orozco-Narváez A, Dávila-Maldonado L, Atisha-Fregoso Y, Diamond B, et al. Serum and cerebrospinal fluid autoantibodies in patients with neuropsychiatric lupus erythematosus. Implications for diagnosis and pathogenesis. PLoS ONE 2008; 3 (10): e3347.

65. Hirohata S, Kanai Y, Mitsuo A, Tokano Y, Hashimoto H, Subcommittee. NR. Accuracy of cerebrospinal fluid IL-6 testing for diagnosis of lupus psychosis. A multicenter retrospective study. Clin Rheuma 2009; 28 (11): 1319-23.

66. Castellino G, Padovan M, Bortoluzzi A, Borrelli M, Feggi L, Caniatti ML, et al. Single photon emission computed tomography and magnetic resonance imaging evaluation in SLE patients with and without neuropsychiatric involvement. Rheumatology (Oxford) 2008; 47 (3): 31923.

67. Driver CB, Wallace DJ, Lee JC, Forbess CJ, Pourrabbani S, Minoshima S, et al. Clinical validation of the watershed sign as a marker for neuropsychiatric systemic lupus 
Lupus neuro-psiquiátrico con déficit cognitivo - T. León et al

erythematosus. Arthritis Rheuma 2008; 59 (3): 332-7.

68. Fitzgibbon BM, Fairhall SL, Kirk IJ, Kalev-Zylinska M, Pui K, Dalbeth N, et al. Functional MRI in NPSLE patients reveals increased parietal and frontal brain activation during a working memory task compared with controls. Rheumatology (Oxford) 2008; 47 (1): 50-3.

69. Jung RE, Caprihan A, Chavez RS, Flores RA, Sharrar J, Qualls CR, et al. Diffusion tensor imaging in neuropsychiatric systemic lupus erythematosus. BMC Neurology 2010; 10 (65).

70. Sibbitt WLJ, Brooks WM, Kornfeld M, Hart BL, Bankhurst AD, Roldan CA. Magnetic resonance imaging and brain histopathology in neuropsychiatric systemic lupus erythematosus. Semin Arthritis Rhaum 2010; 40: 132-52.

71. Jung RE, Segall JM, Grazioplene RG, Qualls C, Sibbitt WL, Roldan CA. Cortical thickness and subcortical gray matter reductions in neuropsychiatric systemic lupus erythematosus. PLoS one 2010; 5 (3): 9302.

72. Buća A, Perković D, Martinović-Kaliterna D, Vlastelica M, Titlić M. Neuropsychiatric systemic lupus erythematosus: diagnostic and clinical features according to revised ACR criteria. Collegium antropologicum 2009;
33 (1): 281-8.

73. Davey R, Bamford J, Emery P. The role of endothelial dysfunction in the pathogenesis of neuropsychiatric systemic lupus erythematosus. Lupus 2010; 19 (7): 797 802.

74. Emmer BJ, Steup-Beekman GM, Steens SC, Huizinga TW, van Buchem MA, van der Grond J. Correlation of magnetization transfer ratio histogram parameters with neuropsychiatric systemic lupus erythematosus criteria and proton magnetic resonance spectroscopy: association of magnetization transfer ratio peak height with neuronal and cognitive dysfunction. Arthritis Rheuma 2008; 58 (5): 1451-7.

75. Bertsias GK, Ioannidis JP, Aringer M, Bollen E, Bombardieri S, Bruce IN, et al. EULAR recommendations for the management of systemic lupus erythematosus with neuropsychiatric manifestations: report of a task force of the EULAR standing committee for clinical affairs. Ann Rheuma Dis 2010; 69 (12): 2074-82.

76. Yazdany J, Yelin E. Health-Related Quality of Life and Employment Among Persons with Systemic Lupus Erythematosus. Rheum Dis Clin North Am 2010; 36 (1): 15-32. 